\title{
Attila Lorincz: Nucleic acid testing for human disease, 2006
}

\author{
Taylor and Francis, hardcover, 470 pages, ISBN: 978-1-57444-543-5
}

\author{
Dr. Matthew C. Fisher
}

(C) Springer-Verlag 2007

The use of nucleic acid tests (NATs) to diagnose human diseases is in the process of revolutionising healthcare. There has been an explosive growth in our knowledge of DNA-sequence variation across the tree of life, driven by rapid technological advances, primarily increases in the speed and cost-effectiveness of DNA sequencing. Concomitantly, NATs have been devised to monitor the relationship between this sequence-diversity and human disease within multiple scenarios. This book is a bold effort to document the current state-of-the-art in NAT diagnosis of major human diseases caused by infections, inherited disorders and cancers. It also includes chapters on the increasing importance and methodological advances for monitoring epigenetic changes and host gene-expression response-profiles.

The book is targeted at research and diagnostic laboratories, as well as interested clinicians, and is broken down into two sections. In Part 1, 'Nucleic Acid Diagnostic Technology', the focus is on the current technologies that underly NATs. Although this is a rapidly developing field, the authors of the individual chapters have covered the breadth of existing techniques (target and signal-amplification-based techniques, in situ hybridization, microarrays and expression profiling) while endeavouring to give some prediction of how future techniques may change current diagnoses. Lorincz has his background in the human papillomavirus field, and this is reflected in Part 2, 'Disease Application of NATs', where there are chapters on detection and differentiation of the major human viral, bacterial

Dr. M. C. Fisher $(\bowtie)$

Department of Infectious Disease Epidemiology,

St Mary's Hospital, London W2 1PG, UK

e-mail: matthew.fisher@imperial.ac.uk and fungal infections. There is inevitably some superfluous data represented in these chapters as the diverse published techniques that are found in the literature rarely mature to useful technologies; a review volume such as this will necessarily cover some techniques that will never become adopted by the research or diagnostic community. For instance, in the diagnosis of fungal infections, NATs have been developed for most taxa; however, there are many issues associated with sensitivity and specificity of the tests that have prevented development of clinical assays; currently the gold-standard for detection remains culturing from clinical specimens. However, the authors do offer opinion as their 'best bet' as to which developments offer most future promise, and what needs to be done to attain these goals. There are also reviews of promising novel applications, such as culture-independent techniques to detect unculturable microbes, as well as the use of host gene expression-response profiles as specific signatures of microbial infections; these techniques offer much for the diagnosis of infection where more traditional methodologies fail. The penultimate chapters of the book focus on the detection of inherited disorders, cancer detection/prognosis and DNA methylation. Due to the existence of more than 4,000 disease-associated genes, these chapters are limited to reviewing methodologies that are associated with detecting and managing the most common disorders. They therefore serve best as an introduction as to what is possible, as well as flagging key challenges that are associated with human genetic testing.

Lorincz and his co-authors have produced a well-organised and comprehensive review of NATs in the twenty-first century. The volume is, to my mind, limited somewhat by a lack of consideration of the ability of NATs to give insights into patterns of evolution of infectious diseases. The spread of drug-resistant determinants through bacterial populations 
is governed by their population genetic structure, and it would have been relevant to include sections on the more common methods that are associated with profiling populations of bacteria, and viruses, such as multilocus sequence testing (MLST) and online databases of gene polymorphisms. Moreover, due to the breadth, and extraordinary pace of developments in the field of NATs, this book serves best as a review of what is possible, rather than offering the last-word. This said, Lorincz and co-authors have produced a comprehensive, useful, and thorough overview of a highly complex field; this book will find a useful place on the shelves of both research and clinical geneticists as well as those developing careers within these fields. 